\title{
A single-arm phase II study of olaparib maintenance with pembrolizumab and bevacizumab in BRCA non-mutated patients with platinum-sensitive recurrent ovarian cancer (OPEB-01): first efficacy results of a 2-stage Simon's design
}

\author{
Jung-Yun Lee', Yoo Na Kim', Sang Wun Kim', Myoung Cheol Lim², Byoung Gie Kim³ , Jae Weon Kim", Hee Seung Kim4, \\ Natalie YI Ngoi ${ }^{5}$, David SP Tan ${ }^{5}$ \\ 'Department of Obstetrics and Gynecology, Yonsei University College of Medicine, Seoul, Korea (jungyunlee@yuhs.ac) \\ ${ }^{2}$ Gynecologic Cancer Branch \& Center for Uterine Cancer, National Cancer Center, Goyang, Korea \\ ${ }^{3}$ Department of Obstetrics and Gynecology, Samsung Medical Center, Sungkyunkwan University School of Medicine, Seoul, Korea \\ ${ }^{4}$ Department of Obstetrics and Gynecology, Seoul National University, Seoul, Korea \\ ${ }^{5}$ Department of Haematology-Oncology, National University Cancer Institute, Singapore
}

Objective: The optimal treatment of BRCA wild-type patients with platinum-sensitive recurrent ovarian cancer remains unknown. Recently, there is an increase in the evidence to support the role of the combination of a poly (adenosine diphosphate-ribose) polymerase inhibitor, anti-angiogenic agents, and immunotherapy as maintenance therapy in BRCA wild-type patients with platinum-sensitive recurrence. We hypothesized that adding pembrolizumab and bevacizumab to olaparib maintenance can increase progression-free survival (PFS) in BRCA wild-type patients with platinum-sensitive recurrent ovarian cancer.

Methods: BRCA wild-type patients who received two previous courses of platinum-containing therapy, achieved complete or partial response to last treatment, and the treatment-free interval is $>6$ months after the penultimate platinum-based chemotherapy offered olaparib maintenance with pembrolizumab and bevacizumab. Forty-four patients will be included from 4 sites across Singapore and Korea. The primary endpoint of the study is 6-month PFS rate. An interim statistical analysis was performed after 22 patients were enrolled. According to Simon's 2-stage optimal design, if 10 or more progressive diseases are observed, the trial will be terminated. If 13 or more non progressive disease at 6 months, the trial will continue to the second stage.

Results: Median age was 60 years old and most of patients (90.9\%) had high-grade serous carcinoma. Secondary debulking operation was performed in $6(27.3 \%)$ patients. The objective response rate (ORR) was $72.7 \%$ and disease control rate (DCR) was $86.4 \%$. At the time of data analysis (data cutoff Nov 6 2021), 13 had non-progressive disease at 6 months. No grade 4 adverse events were reported and no treatment related adverse events leading to treatment discontinuation were observed. Additional correlative data regarding homologous recombination deficiency will be presented at the meeting.

Conclusion: This is the first report of maintenance therapy with triplet combination in ovarian cancer. Based on the efficacy result of the interim analysis, it is worth moving on to the second stage. These combinations showed a manageable safety profile. 